\title{
Free energies of crystalline solids: a lattice-switch Monte Carlo method
}

\author{
A.D. Bruce, N.B. Wilding \& G.J. Ackland \\ Department of Physics and Astronomy, The University of Edinburgh \\ Edinburgh, EH9 3JZ, Scotland, United Kingdom
}

\begin{abstract}
We present a method for the direct evaluation of the difference between the free energies of two crystalline structures, of different symmetry. The method rests on a Monte Carlo procedure which allows one to sample along a path, through atomic-displacement-space, leading from one structure to the other by way of an intervening transformation that switches one set of lattice vectors for another. The configurations of both structures can thus be sampled within a single Monte Carlo process, and the difference between their free energies evaluated directly from the ratio of the measured probabilities of each. The method is used to determine the difference between the free energies of the $f c c$ and $h c p$ crystalline phases of a system of hard spheres.
\end{abstract}

PACS numbers: 64.70Kb, 02.70.Lq, 71.20Ad 
One of the fundamental tasks of theoretical condensed matter physics is to understand the observed structures of crystalline materials in terms of microscopic models of the atomic interactions. The principles involved are well known: one needs to evaluate which of the candidate structures has the lowest free energy for given (model and thermodynamic) parameters. In practice the task is rather less straightforward. Conventional Boltzmann importance sampling Monte Carlo (MC) methods do not yield the free energy [1]. It is therefore customary to resort to integration methods (IM) which determine free energies by integrating free-energy derivatives measured at intervals along a parameter-space-path connecting the system of interest to a reference system whose free energy is already known. This procedure has been used widely, and with ingenuity [2]. Nevertheless it leaves much to be desired. In particular, to determine the difference between the free energies of two phases one has to relate each of them separately to some reference system, with uncertainties which are not always transparent, and which can be significant on the scale of the free energy difference of interest. Clearly, one would prefer a method which focuses more directly on this difference. The elements of such a strategy are to be found in the extendedsampling techniques pioneered by Torrie and Valleau [3], and recently revitalized in the multicanonical method of Berg and Neuhaus 4. The key concept underlying this method is that of a configuration-space-path comprising the macrostates of some chosen macroscopic property $\mathcal{M}$. The method utilizes a sampling distribution customized to even out the probabilities of different $\mathcal{M}$-macrostates. In principle, it allows one to sample along a path (whose canonical probability is generally extremely small) chosen to connect the distinct regions of configuration space associated with two phases; the difference between the free energies of the two phases can then be obtained directly from the ratio of the probabilities with which the system is found in each of the two regions. This idea has been applied in the investigation of the phase behavior of ferromagnets [4], fluids [5], and lattice gauge theories [6]. However its application to structural phase behavior faces a distinctive problem: finding a path that links the regions of configuration space associated with two different crystal structures [7], without traversing regions of non-crystalline order, which present problems [8] for even multicanonical Monte-Carlo studies. We show here how one may construct such a path, and use it for direct high-precision measurement of free-energy differences of crystal structures.

The idea is simple; we describe it first in general and qualitative terms. The atomic position coordinates are written, in the traditions of lattice dynamics, as the sum of a lattice vector [9], and a displacement vector. The configurations associated with a particular structure are explored by MC sampling of the displacements. Given any configuration of one structure one may identify a configuration of the other, by switching one set of lattice vectors for the other, while keeping the displacement vectors fixed. Such lattice switches can be incorporated into the MC procedure by regarding the lattice type as a stochastic variable. Lattice switches have an intrinsically low acceptance probability, since typically they entail a large energy cost. But the multicanonical method can be used to draw the system along a path comprising the macrostates of this 'energy cost', and thence into a region of displacement-space in which the 'energy cost' is low, and the lattice switch can be implemented. The net result is a MC procedure which visits both structures in the course of a single simulation, while never moving out of the space of crystalline configurations. The method is, we believe, potentially very general. We illustrate it here by using it to determine the difference between the free energies of the two close-packed structures ( $f c c$ and $h c p$ ) of a system of hard spheres. This problem has a long history [10]. The difference between the free energies (effectively, the entropies) is extremely small, and

recent IM studies have disagreed on its value [11,12]. It thus provides an exacting and topical testing ground for the method proposed here [13].

We consider a system of $N$ particles with spatial coordinates $\{\vec{r}\}$. The particles are confined in a fixed volume $V$, with periodic boundary conditions [14]. We make the decomposition

$$
\vec{r}_{i}=\vec{R}_{i}+\vec{u}_{i}
$$

where the vectors $\vec{R}_{i}, i=1 \ldots N \equiv\{\vec{R}\}_{\alpha}$ define the sites of a lattice of type $\alpha$ (here, either $f c c$ or $h c p$ ). Clearly there are many transformations that will map one set of vectors into the other; the mapping we have chosen is explained in Fig. 1(a),(b): it exploits the fact that the two structures differ only in the stacking pattern of the close-packed planes.

We define a partition function (and free energy) associated with the structure $\alpha$ by 15$]$

$$
\begin{aligned}
Z(N, V, T, \alpha) & =\int_{\{\vec{u}\} \in \alpha} \prod_{i}\left[d \vec{u}_{i}\right] \exp [-\Phi(\{\vec{u}\}, \alpha)] \\
& \equiv \exp \left[-F_{\alpha}(N, V, T) / k T\right]
\end{aligned}
$$

where $\Phi$ represents the dimensionless configurational energy. In the present context

$$
\Phi(\{\vec{u}\}, \alpha) \equiv \Phi(\{\vec{r}\})= \begin{cases}0 & \left|\vec{r}_{i}-\vec{r}_{j}\right|>\sigma \forall i, j \\ \infty & \text { otherwise }\end{cases}
$$

where $\sigma$ is the hard sphere diameter. The $\alpha$-label attached to the integral in Eq. (2) signifies that it must include only contributions from configurations within the subspace associated with the structure $\alpha$ [16.

Consider now the canonical ensemble with probability distribution

$$
P(\{\vec{u}\}, \alpha \mid N, V, T)=\frac{\exp [-\Phi(\{\vec{u}\}, \alpha)]}{Z(N, V, T)}
$$


where $Z(N, V, T) \equiv \sum_{\alpha} Z(N, V, T, \alpha)$. The probability that the system will be found to have structure $\alpha$ provides a measure of the associated partition function:

$$
\begin{aligned}
P(\alpha \mid N, V, T) & \equiv \int_{\{\vec{u}\} \in \alpha} \prod_{i}\left[d \vec{u}_{i}\right] P(\{\vec{u}\}, \alpha \mid N, V, T) \\
& =\frac{Z(N, V, T, \alpha)}{Z(N, V, T)}
\end{aligned}
$$

The difference between the free-energies of the two structures may be thus be expressed as

$$
F_{h c p}(N, V, T)-F_{f c c}(N, V, T) \equiv N k T \Delta f=k T \ln \mathcal{R}
$$

where,

$$
\mathcal{R}=\frac{Z(N, V, T, f c c)}{Z(N, V, T, h c p)}=\frac{P(f c c \mid N, V, T)}{P(h c p \mid N, V, T)}
$$

This identification is useful only if one can devise a MC procedure that will actually visit the configurations $\{\vec{u}\}, \alpha$ with the probabilities prescribed by Eq. (何). To do so one must deal with the ergodic block against lattice switches ('updates' of the lattice label, $\alpha$ ): almost invariably such a switch maps an accessible configuration of one structure onto an inaccessible configuration of the other (one which violates the hard-sphere constraint implied by Eq. (3)). Fig. 1(b) provides an example. The resolution is to bias the sampling procedure so as to favor the occurrence of configurations which transform without violating this constraint. To do so we define an overlap order parameter

$$
\mathcal{M}(\{\vec{u}\}) \equiv M(\{\vec{u}\}, h c p)-M(\{\vec{u}\}, f c c)
$$

where $M(\{\vec{u}\}, \alpha)$ counts the number of pairs of overlapping spheres associated with the configuration $\{\vec{u}\}, \alpha$ (again, see Fig. 1(b)). Since $M(\{\vec{u}\}, \alpha)$ will necessarily be zero for any set of displacements $\{\vec{u}\}$ actually visited when the system has lattice $\alpha$, the order parameter $\mathcal{M}$ is necessarily $\geq 0(\leq 0)$ for realizable configurations of the $f c c(h c p)$ structure. The displacement configurations with $\mathcal{M}=0$ are accessible in both structures and thus offer no barrier against lattice switches. Accordingly the set of $\mathcal{M}$-macrostates provides us with the required 'path' connecting the two phases, through a lattice-switch at $\mathcal{M}=0$. To pick out this path we must sample from the biased configuration distribution

$$
P(\{\vec{u}\}, \alpha \mid N, V, T,\{\eta\}) \propto P(\{\vec{u}\}, \alpha \mid N, V, T) e^{\eta(\mathcal{M}(\{\vec{u}\}))}
$$

where $\{\eta\} \equiv \eta(\mathcal{M}), \mathcal{M}=0, \pm 1, \pm 2 \ldots$ define a set of multicanonical weights [4], which have to be determined such that configurations of all relevant $\mathcal{M}$-values are sampled. Once this is done, one can measure the weighted distribution of $\mathcal{M}$-values, and reweight (unfold the bias) to determine the true canonical form of this distribution:

$$
P(\mathcal{M} \mid N, V, T) \propto P(\mathcal{M} \mid N, V, T,\{\eta\}) e^{-\eta(\mathcal{M})}
$$

Finally, the difference between the free energies of the two structures may be read off from this distribution through the identification (cf Eqs. (6), (7) ) $\Delta f=N^{-1} \ln \mathcal{R}$, with

$$
\mathcal{R}=\frac{\sum_{\mathcal{M}>0} P(\mathcal{M} \mid N, V, T)}{\sum_{\mathcal{M}<0} P(\mathcal{M} \mid N, V, T)}
$$

We have implemented this procedure to study systems of $\mathrm{N}=216,1728$ and 5832 hard spheres (forming, respectively 6,12 or 18 close-packed layers). The volume $V$ was chosen such that the fraction of space filled, $\rho$, satisfies $\rho / \rho_{c p}=0.7778$ [17, where $\rho_{c p} \equiv 0.7404$ is the space filling fraction in the closest packing limit. The MC procedure entails sampling the displacement variables $\{\vec{u}\}$ and the lattice label $\alpha$. The variables $\{\vec{u}\}$ were updated by drawing new values from a top-hat distribution [18] and accepting them provided they satisfy the hard sphere constraint; the lattice switches were attempted (and accepted with probability $1 / 2$ ) only when the system is in the $\mathcal{M}=0$ macrostate. The weights (which enable the system to reach this special macrostate) were obtained using methods explained elsewhere [7]. We allowed typically $2 \times 10^{4}$ sweeps for equilibration and up to $5 \times 10^{7}$ sweeps for final sampling runs on the largest system size. The simulations were conducted on DEC ALPHA workstations using overall some 800 hours CPU time.

Fig. 2 shows the measured overlap distribution for the system of $N=1728$ spheres; the inset shows the probability on a logarithmic scale, exposing the enormity of the entropic 'barrier' (probability 'trough') that the multicanonical weighting enables us to negotiate. The difference between the free energies of the two structures is identifiable immediately and transparently from the ratio of the integrated weights of the two essentially gaussian peaks. Our results for this system and other system sizes are gathered together in Table 1, along with the results of other authors.

From Table 1 it is apparent that the present work greatly refines the largely inconclusive results of the original IM study [2]. Our results are consistent with -though substantially more precise than- very recent IM studies of Bolhuis and Frenkel 12]. They are inconsistent with the result reported by Woodcock [11], given that $\Delta f$ is believed to decrease as the density is reduced, towards melting [19]. While we have not attempted an explicit analysis of the finite-size behavior, the close agreement between our results for $N=1728$ and $N=5832$ indicates that the latter should provide an extremely good estimate of the thermodynamic limit, confirming the stability of the $f c c$ structure at this density.

Our principal concern here, however, is with the general lessons that can be learned about the method introduced in this work. The precision we have achieved with this method is self-evidently a significant advance on that of IM studies. Admittedly, this level of precision has entailed substantial processing time, principally because of 
the relative slowness of the diffusive exploration of the multicanonically weighted configuration space. But the point is that the procedure is practicable [20], with a computational strategy that is, we suggest, less complex and more transparent than that of IM. Thus, for example, the method described in [2] involves integration (of a meansquare displacement) along a parameter-space-path connecting each structure to a reference system, comprising an Einstein-model of the same structure; the MC integral then has to be combined with the known free energy of the Einstein model, a virial correction, and a correction to the virial correction, before taking the difference between the results for the two structures. The uncertainties in all the contributions have to be assessed separately. By contrast, the present method focuses directly on the quantity of interest (the relative weights of the peaks in Fig. 2); and the precision with which it is prescribed is defined by standard MC sampling theory.

Finally we comment briefly on the more general applicability of the method. For systems other than hard spheres, the role of the overlap order parameter is played by the energy barrier encountered in the lattice switch; the generalization of the weighting procedure should be straightforward. It seems unlikely that many problems will require the level of precision needed here, where the two phases are so finely balanced. However some circumstances will not generally prove as favorable. In the present case one can readily identify a lattice-to-lattice mapping which guarantees no overlaps (high-energy-cost interactions) amongst subsets of the atoms (those lying within the same close-packed plane). The optimal form of mapping may not always be so evident. It may also prove advantageous to relax the constraint imposed in Eq. (1) that the coordinates $\{\vec{u}\}$ represent identical displacement patterns in the two structures. At the cost of only little extra computational complexity the true displacements may be represented as structure-dependent (even site-dependent) functions of a (still common) set of coordinates $\{\vec{u}\}$. It should then be possible to ensure that a typical displacement pattern in one structure maps onto a typical pattern in the other. These matters are the subject of ongoing study.

[1] K. Binder, J. Comp. Phys. 591 (1985)

[2] D. Frenkel \& A.J.C. Ladd, J. Chem. Phys 81, 3188 (1984).

[3] G.M. Torrie \& J.P. Valleau, Chem. Phys. Lett. 28, 578 (1974).

[4] B.A. Berg \& T. Neuhaus, Phys. Lett. B 267, 249 (1991); Phys. Rev. Lett. 68, 9 (1992).

[5] N.B. Wilding, Phys. Rev. E 52,602 (1995).

[6] B. Grossmann, M.L. Laursen, T. Trappenberg \& U.J. Wiese, Phys. Lett. B 293, 175 (1992).
[7] G.R. Smith \& A.D. Bruce, Phys. Rev. E 53, 6530 (1996) apply multicanonical methods to a structural phase transition which involves no change of symmetry where an appropriate path is identified simply by $\mathcal{M}=\rho$.

[8] Eg: the ergodic block associated with re-crystallization.

[9] We use the term 'lattice vector' a little loosely: we mean the set of vectors identified by the orthodox crystallographic lattice, convolved with the orthodox basis.

[10] B.J.Alder, B.P. Carter \& D.A. Young, Phys Rev 183, 831 (1969).

[11] L.V. Woodcock, Nature 384, 141 (1997).

[12] P.G.Bolhuis \& D. Frenkel (unpublished).

[13] The hard sphere system has a wider significance. Y.Choi et al,J. Chem. Phys. 999917 (1993) show that predictions for the phase diagram of a Lennard Jones solid depend extremely sensitively on the hard-sphere free-energy difference $\Delta f$ computed here. Colloids provide experimental realizations of near-hard-sphere systems: P.N. Pusey et al, J. Phys: Condens. Matter 6 A29 (1994).

[14] In common with previous studies we work at constant volume, and constrain the $c / a$ ratio in the $h c p$ structure to its closest-packing value. Generalization to the constant pressure ensemble is straightforward in principle.

[15] We use a general notation; formally the properties of the hard-sphere system are independent of $T$.

[16] In the MC context the configurations associated with a given structure are identified as the set which are actually accessed in a simulation initialized within the set.

[17] This value of $\rho$ was chosen to coincide with one of those studied in Ref [2].

[18] This choice of sampling procedure ensures that the center of mass is effectively fixed. For consistency the width of the top-hat distribution must be large compared to the range of displacements actually accepted.

[19] This is the implication of studies of the pressure in the two structures: B.J.Alder, D.A. Young, M.R. Mansigh \& Z.W. Salsburg, J. Comp. Phys 7, 361 (1971).

[20] The time required is measured on a scale of hours rather than the eons required if one were to attempt such a 'direct' method without the multicanonical strategy provided here: recall the scale on the inset of Fig. 2. 


\begin{tabular}{|lrrrr}
\hline \hline$\rho / \rho_{c p}$ & $\mathrm{~N}$ & \multicolumn{2}{c}{$\Delta f \times 10^{5}$} & Ref. \\
\hline 0.7360 & 216 & 90 & $(135)$ & 2 \\
0.7360 & 12000 & 500 & $(100)$ & 11 \\
0.7360 & 12906 & 90 & $(20)$ & 12 \\
0.7778 & 1152 & -120 & $(180)$ & $2]$ \\
0.7778 & 216 & 101 & $(4)$ & $\mathrm{PW}$ \\
0.7778 & 1728 & 83 & $(3)$ & $\mathrm{PW}$ \\
0.7778 & 5832 & 86 & $(3)$ & $\mathrm{PW}$ \\
\hline \hline
\end{tabular}

TABLE I. Results for the difference between the free energy of $h c p$ and $f c c$ structures, as defined in Eq. (6) with associated uncertainties in parentheses. Results attributed to Ref. [2 were deduced by combining the separate results for $f c c$ and $h c p$ given there. PW signifies the present work. The PW error bounds were computed from the statistical uncertainties in the weights of the peaks in $P(\mathcal{M})$.

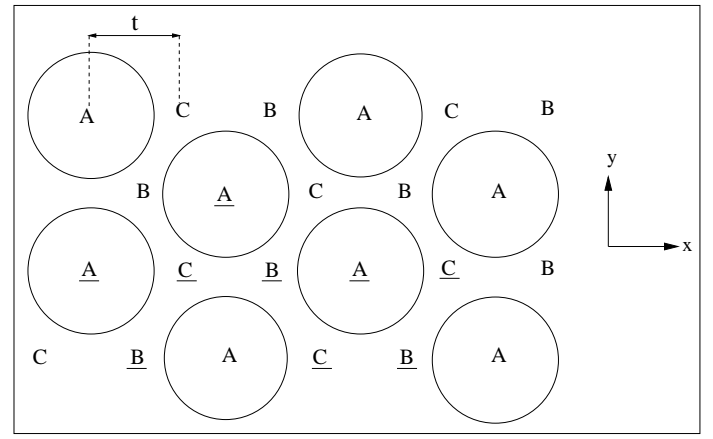

FIG. 1a. Schematic representation of the close-packed structures. The points marked A show the positions of the sites in one close-packed $(x y)$ layer; the circles show the boundaries of spheres occupying these sites in an ideal (zero-displacement) structure. The points marked B and C show the projections of sites in other layers (stacked along the $z$-axis) onto the $x y$ plane; the $f c c$ and $h c p$ structures entail sequences of type $A B C A \ldots$ and $A B A B \ldots$ respectively. The lattice switch from $f c c$ to hcp entails translations of the close-packed planes, as detailed in Fig. 1(b).

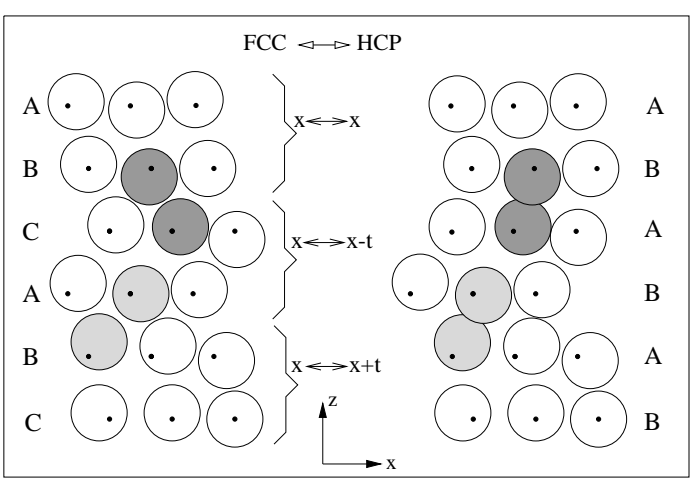

FIG. 1b. [Left side] The positions of the spheres in an arbitrary configuration of the fcc structure, projected onto the $x z$ plane. We show 6 layers, with 3 spheres in each; the sites in the top 3 layers $(\mathrm{A}, \mathrm{B}, \mathrm{C})$ correspond to those marked (and underlined) A,B,C in Fig. 1(a).

[Center] The action of the lattice switch: reading from the top, the first two layers $(\mathrm{A}, \mathrm{B})$ of the $f c c$ structure are invariant; the next two $(\mathrm{C}, \mathrm{A})$ layers are translated along the $x$ direction by $-t$; and the final two $(\mathrm{B}, \mathrm{C})$ layers are translated by $+t$, where $t$ is identified in Fig. 1(a).

[Right side] Projections of the spheres in the resulting hcp arrangement. Here, the displacements $\{\vec{u}\}$, realizable in the $f c c$ structure, give two overlapping pairs of spheres (shaded) in the $h c p$ structure so that $\mathcal{M}(\{\vec{u}\})=2$ in this case. Note that the picture is schematic: in particular, the density shown here is much lower than that chosen for the present study.

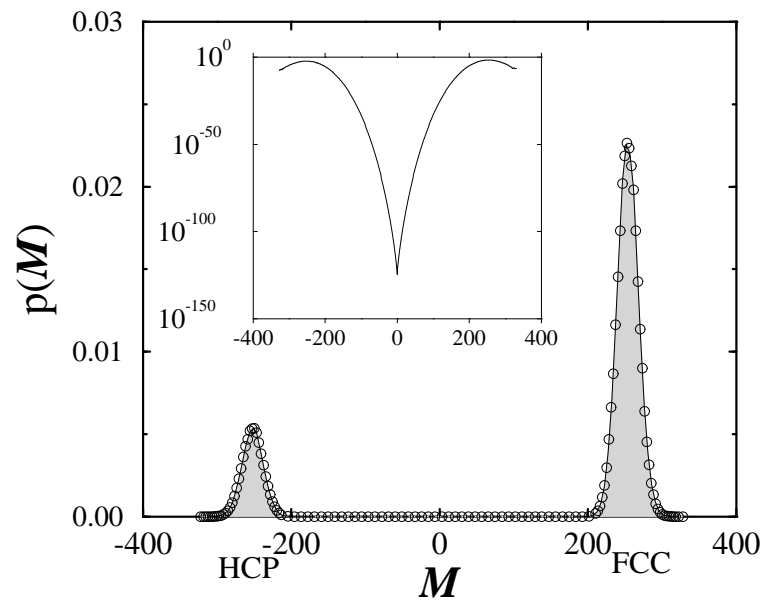

FIG. 2. The distribution of the overlap parameter $\mathcal{M}$ for a system of $\mathrm{N}=1728$ spheres; the inset shows the distribution on a logarithmic scale. The statistical uncertainties are smaller than the symbol size. The free-energy difference $\Delta f$ is identified from the logarithm of the ratio $\mathcal{R}$ of the weights of the two peaks (Eq.(11)). The smallness of $\Delta f$ allows both peaks to be displayed on one linear scale in this case. 